\title{
Ehlers-Danlos syndrome type IV: phenotypic consequences of a splicing mutation in one COL3A1 allele
}

\author{
D O Sillence, A A Chiodo, P E Campbell, W G Cole
}

\begin{abstract}
The features of a child with Ehlers-Danlos syndrome type IV (EDS IV) resulting from a mutation in one COL3A1 allele were studied. The child was heterozygous for a G- to Atransition at the splice donor site of intron 41 . It resulted in the splicing out of the exon 41 encoded sequence from $\alpha 1$ (III) mRNA and the deletion of 36 amino acids from glycine ${ }^{775}$ to lysine $^{810}$ of the triple helical domain of $\alpha 1$ (III) chains of type III collagen. The amount of type III collagen in the dermis was only about $11 \%$ of normal. The child had the acrogeric form of EDS IV. He had the characteristic facies with a pinched nose, thin lips, and prominent eyes. These facial features, his aesthenic build, thin skin, prominent subcutaneous veins, and aged hands produced a 'cachectic' appearance. These features were evident in early childhood and worsened up to $12 \frac{1}{2}$ years when he was last reviewed. Spontaneous bruising, bleeding from the large bowel, constipation, and delayed gastric emptying were other features. In cross section, the dermal collagen fibrils were round and measured $93.3 \pm 11.5 \mathrm{~nm}$ in diameter which was not significantly different from control values of $102 \cdot 5 \pm 13.4 \mathrm{~nm}$. The serum type III procollagen amino-terminal propeptide level of $25.5 \mathrm{ng} / \mathrm{ml}$ was within the normal age matched values of $15.5 \pm 7 \cdot 7 \mathrm{ng} / \mathrm{ml}$ despite the low production of type III collagen
\end{abstract}

Medical Genetics and Dysmorphology Unit, Children's Hospital, Camperdown, NSW 2050, Australia. D O Sillence

Department of Paediatrics, University of Melbourne, Australia.

A A Chiodo, W G Cole

Department of Anatomical Pathology, The Royal Children's Hospital, Parkville, Australia.

P E Campbell

Correspondence to Professor Sillence.

Received for publication 2 May 1991.

Accepted for publication 17 May 1991. by cultured fibroblasts. The child probably had a spontaneous new mutation in one COL3A1 allele as only normal sequences were obtained from the corresponding amplified region of the parent's leucocyte DNA.

The Ehlers-Danlos type IV syndrome (EDS IV) is a heterogeneous group of disorders resulting from abnormalities in type III collagen. ${ }^{1}$ Unlike the other types of EDS, joint hypermobility is restricted to the small joints of the hands and hyperelasticity of the skin is largely confined to the dorsal aspect of the elbows and the sides of the neck. Severe bruising and arterial fragility also set EDS IV apart from the other Ehlers-Danlos syndromes. ${ }^{1-5}$ The recognition that the severity of EDS IV varies widely, from almost insignificant disease through to an extremely severe disease with episodes of life threatening ecchymosis and vascular rupture, has focused attention on a group of patients whose underlying disorder may not be readily apparent when joint hypermobility and cutaneous hyperelasticity are used as the sole diagnostic criteria of the Ehlers-Danlos syndrome. ${ }^{4-6}$

In this paper we describe the phenotypic consequences of a previously reported COL3A1 mutation in a child with EDS IV. ${ }^{7}$ The child was shown to be heterozygous for a GT- to AT- transition at the splice donor site of intron 41 of the COL3Al gene. This mutation generated an abnormally spliced mRNA in which sequences of exon 40 and 42 were joined together with maintenance of the reading frame. Deletion of the 108 nucleotides encoded by exon 41 resulted in the deletion of 36 amino acids, from glycine ${ }^{775}$ to lysine $e^{810}$ of the triple helical domain of the $\alpha 1$ (III) chain of type III collagen. The peptide deletion included the mammalian collagenase cleavage site at glycine ${ }^{781}$-isoleucine ${ }^{782}$, which accounted for the resistance of the mutant type III collagen to mammalian collagenase digestion. Cultured dermal fibroblasts produced a reduced amount of type III collagen which was secreted poorly. The molecules included normal homotrimers, mutant homotrimers, and mixed heterotrimers. However, the dermis only contained about $11 \%$ of the normal amount of type III collagen. 


\section{Case report}

The proband was the third child of his 26 year old mother. His father was aged 24. His unrelated parents did not show any clinical features of EDS IV and there was no family history of the disease. He was born at term after a normal vaginal delivery. The birth weight was $2800 \mathrm{~g}$ (50th centile) and length $54.5 \mathrm{~cm}$ (50th centile).

Poor appetite and weight gain were observed from birth and he had a 'cachectic' appearance from early childhood (fig 1). He had a flat midface with a pinched nose and thin lips. His longitudinal growth was maintained on the 10th to 25th centile and his weight gain followed the 3 rd to 10 th centile. His 'cachectic' appearance and the presence of multiple bruises resulted in the diagnosis of 'child abuse' on many occasions before the diagnosis of EDS IV was made at 5 years of age.

The first abnormality noted by the parents was bright bleeding from the rectum at 9 weeks of age. Fresh and old blood were separate from the stool and sometimes mixed with it such that the bleeding was presumed to be coming from the lower gastrointestinal tract. This proposal was confirmed at 2 years of age by colonoscopy which showed fresh red blood in the rectum and very friable rectal mucosa. Bleeding from the rectum continued intermittently for three years and was not associated with constipation. In later childhood he was constipated with bowel actions once every three to four days. It was unrelieved by liquid paraffin, psyllium hydrophilic mucilloid, or domperidone.

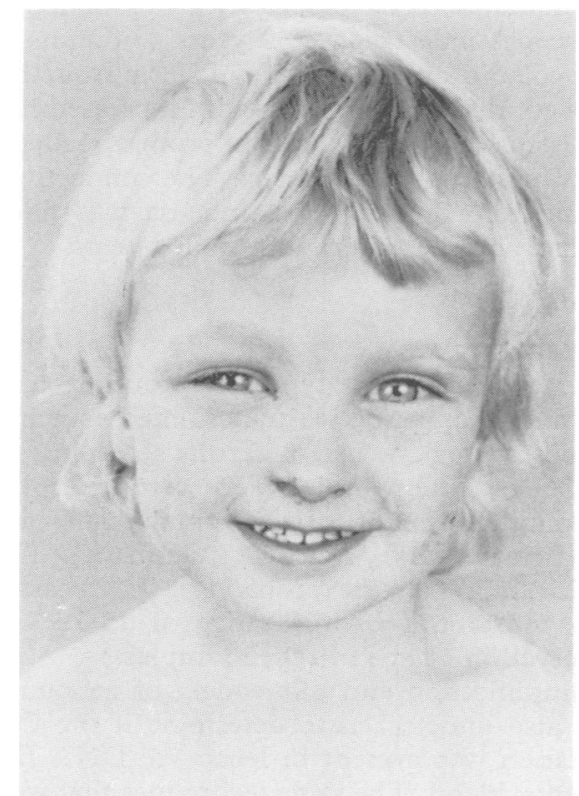

Figure 1 Facial appearance at 2 years 3 months of age showing a pinched nose and thin lips.
Bruising on the trunk and limbs occurred spontaneously from 10 months of age. While blunt trauma led to severe bruising, there was no history of life threatening ecchymoses or haemarthrosis. The cause of his easy bruising was extensively investigated at five different medical centres before the diagnosis of EDS IV was made and no abnormality of coagulation or platelet function was found.

The skin on the ventral surface of his body and limbs was soft, thin, and translucent, and the underlying veins were prominent. The skin over his lower abdomen was finely wrinkled. These features were first noted in infancy. Cutaneous hyperelasticity was only present at the base of the neck and behind both elbows. The skin on the dorsum of the hands was finely wrinkled and prematurely aged at 2 years 3 months (fig 2). The skin became more atrophic and violaceous with age.

Skin fragility and poor wound healing were not apparent. Minor lacerations on his shins had healed normally. Two lacerations required suturing and they also healed without dehiscence or abnormal scarring.

$\mathrm{He}$ was re-examined at the age of $12 \frac{1}{2}$ years. $\mathrm{He}$ still had spontaneous bruising and constipation. The physical findings were similar to those observed in early childhood but were more marked. There were dark rings around his eyes which were deeply set and he had a pinched nose and thin lips. These facial features and his aesthenic build gave him a 'cachectic' appearance which was more marked than in early childhood (fig 3). His height was $140 \mathrm{~cm}$ (10th centile), arm span $143 \mathrm{~cm}$, and upper segment/lower segment ratio 0.97 .

The hyperelasticity of the skin was still confined to the neck and elbow skin. There was no scarring of the forehead or knees. There were no inguinal herniae and the testes were fully descended. His elbows and wrists were hypermobile but the range of movement in his fingers, knees, and ankles was normal. He had postural flat feet. There was no clinical spinal deformity.

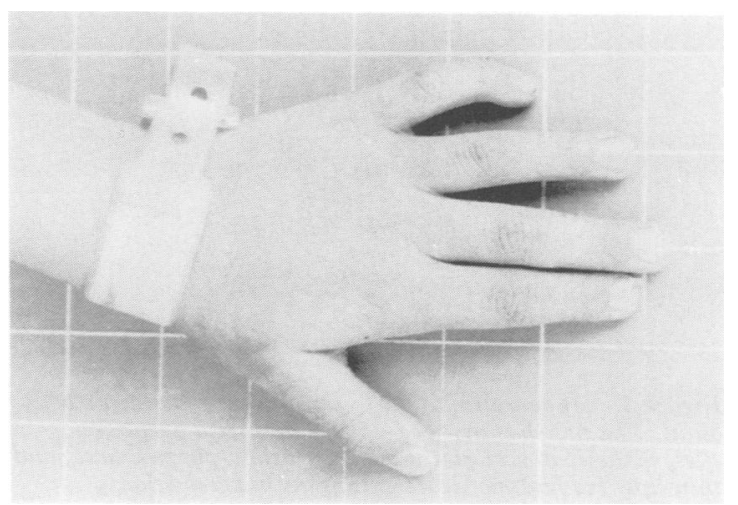

Figure 2 . Hand at the age of 2 years 3 months showing a finely wrinkled, aged appearance. 
Dental examination showed a normal class I occlusion with rotation of the upper lateral incisors.

\section{INVESTIGATIONS}

Coagulation studies were performed on numerous occasions. At 5 years of age, the skin bleeding time was mildly increased to 13 minutes (normal range 2.0 to 9.5 minutes). The platelet count was normal and platelets aggregated normally with ADP, adrenalin, collagen, and ristocetin. Factor VIII Rag and Von Willebrand factor levels were normal.

A skeletal survey at $12 \frac{1}{2}$ years showed relatively slender long bones, minimal platyspondyly of the lower lumbar vertebrae, and minor scoliosis in the lumbar spine. The hands did not show acro-osteolysis. ${ }^{1}$ His bone age was within normal limits. A CT scan of his head, undertaken at 5 years of age because his head circumference was $46 \mathrm{~cm}$ (less than the $3 \mathrm{rd}$ centile), was normal.

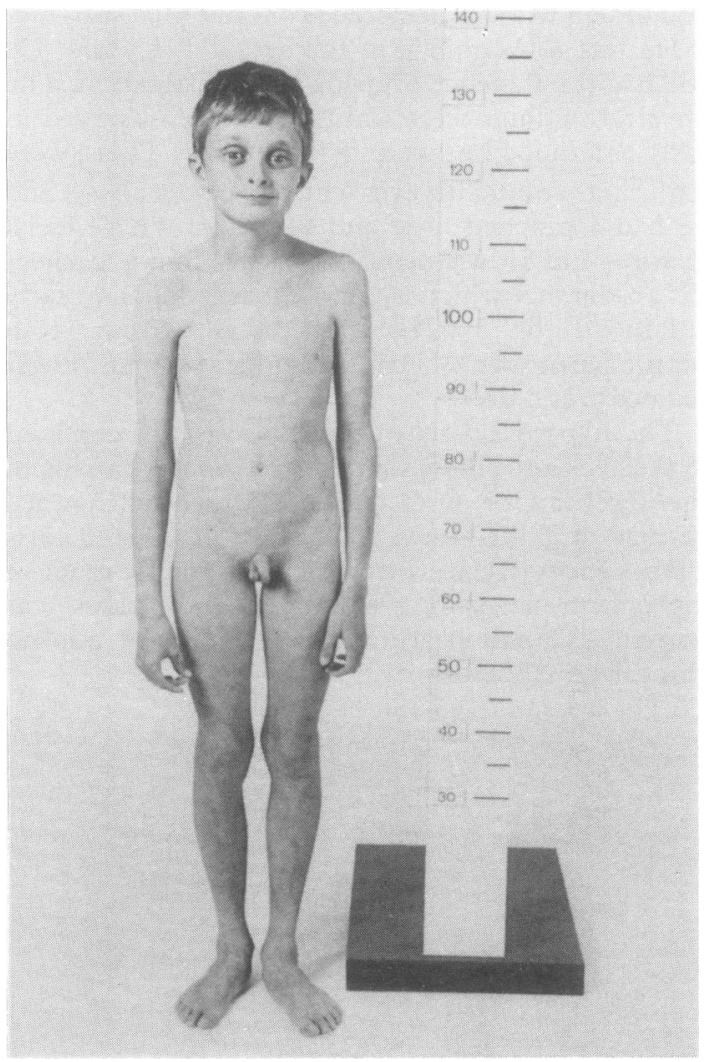

Figure 3 Appearance at $12 \frac{1}{2}$ years showing aesthenic body build. The face has an aged appearance with deeply set eyes, violaceous periorbital discolouration, pinched nose, and thin lips. He had moderate cutaneous hyperelasticity, particularly of the neck and elbows. The hands have an aged appearance with atrophic and transparent skin. There was minimal scarring. There was mild distal joint laxity.
Echocardiography with 2D Doppler scanning in the ' $M$ ' mode showed that the heart, heart valves, and major blood vessels were normal. A Doppler abdominal ultrasound also showed that the liver, pancreas, gall bladder, and kidneys were normal.

While a barium meal and follow through examination did not show any abnormality, a nuclear medicine gastric emptying study showed slow movement of the tracer from the stomach to the small bowel with a clearance $\left(t_{1}\right)$ of 128 minutes (normal $=37 \pm 5$ minutes).

\section{SKIN HISTOLOGY}

A punch biopsy was obtained from his thigh at 5 years 3 months for electron microscopy. The skin was soft and thin and the biopsy hole immediately retracted to produce an abnormally large defect which bled profusely.

The biopsy was processed for electron microscopy as previously described. ${ }^{8}$ The dermal fibroblasts appeared to be normal (fig 4). The mid-dermal collagen fibril cross sectional profiles and elastin structure and staining were within normal limits (fig 5 ). The mean cross sectional collagen fibril diameter was $93.3 \pm 11.5 \mathrm{~nm}(\mathrm{n}=390)$. The mean fibril diameter was marginally smaller than the mean value of $102.5 \pm 13.4 \mathrm{~nm}(\mathrm{n}=1000)$ obtained from a closely age matched control sample of inguinal skin.

\section{INTESTINAL HISTOLOGY}

Colonic and rectal biopsies were obtained at colonoscopy undertaken at 2 years 4 months of age. The colonic biopsies, at 15 and $25 \mathrm{~cm}$ from the anus, showed mild inflammation with increased numbers of plasma cells in the lamina propria, oedema, and areas of attenuated epithelia. The connective tissue of the colonic and rectal mucosa was finer than normal and there were thin walled veins.

\section{BIOCHEMISTRY}

The level of serum type III procollagen aminoterminal propeptide was determined as it has been proposed to be a useful serum screening test for EDS IV. ${ }^{\text {The }}$ Theptide levels were determined by radioimmunoassay using a commercially available kit (Behringwerke, Marburg, Germany). The level of $25.5 \mathrm{ng} / \mathrm{ml}$ in the proband at $12 \frac{1}{2}$ years of age was within the normal range of $15.5 \pm 7.7 \mathrm{ng} / \mathrm{ml}$ observed in 15 age matched samples.

Amplification and sequencing of genomic DNA was also undertaken to determine if the COL3A1 mutation was present in leucocyte DNA from the parents' blood. The same techniques that were used to define the genomic DNA mutation in the proband were used. ${ }^{7}$ Only normal DNA sequences were 


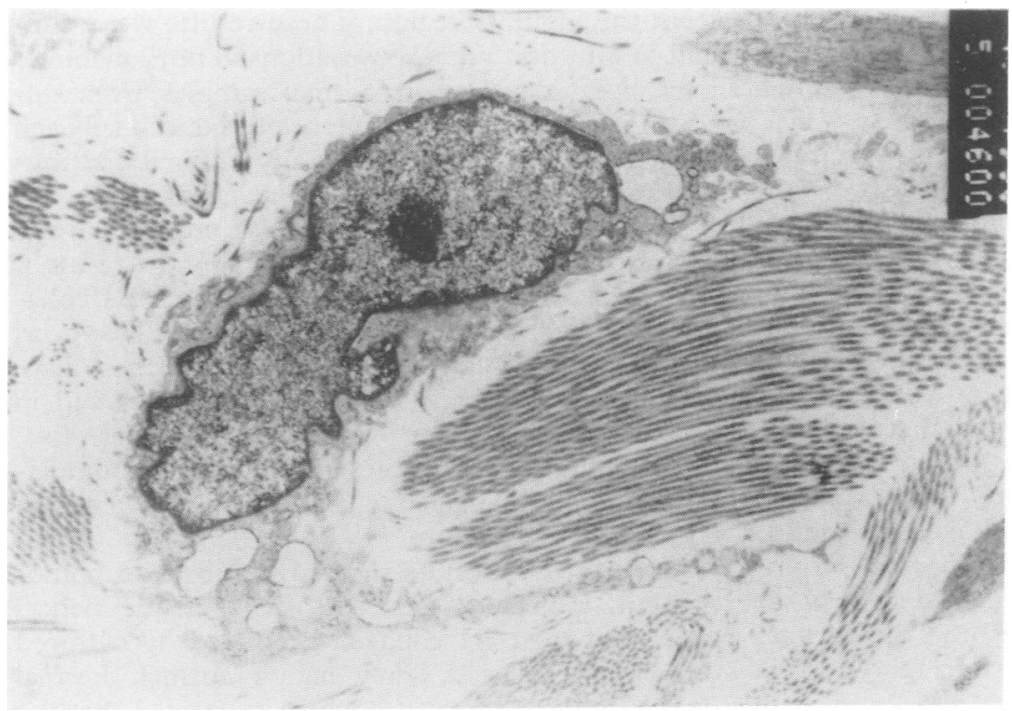

Figure 4 Dermal fibroblast with normal rough endoplasmic reticulum and collagen fibrils in longitudinal cross section.

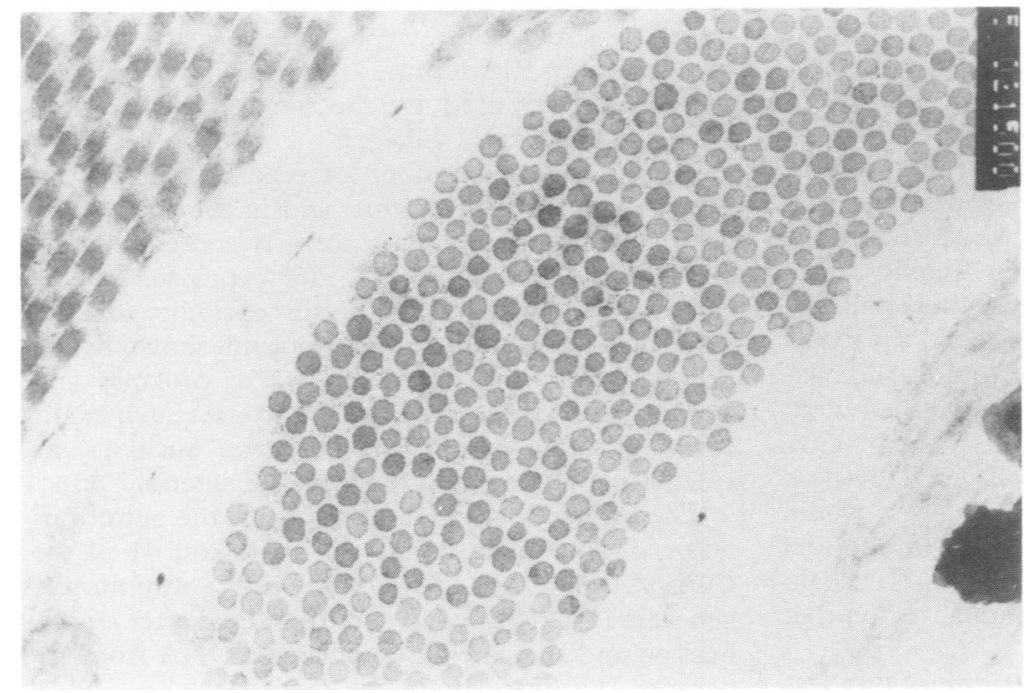

Figure 5 Dermal collagen fibre bundle with normal cross sectional fibril pattern.

obtained from the polymerase chain reaction products. The sequence extended from exon 41 to exon 42 of the COL $3 A 1$ gene and included the splice donor site of intron 41 , the site of the mutation in the proband. ${ }^{7}$ Neither parent showed any evidence of the mutant sequence in the amplification products.

\section{Discussion}

The proband had the typical features of the acrogeric form of EDS IV. ${ }^{1} \mathrm{He}$ had the characteristic facies with a pinched nose, thin lips, prominent eyes, and violaceous eye lids. These facial features, his aesthenic build, thin skin, prominent subcutaneous veins, and acrogeric hands produced a 'cachectic' appearance. These features were evident in early childhood and progressively worsened up to the time of review at $12 \frac{1}{2}$ years of age.

His failure to thrive, bleeding from the rectum, and frequent bruising were suspected initially to be the result of 'child abuse'. The proband did not have the marked joint hypermobility and cutaneous hyperelasticity that are characteristic of most types of EDS. ${ }^{10}$ The numerous hypoplastic scars on the 
forehead, elbows, knees, and shins in EDS types I and II were not present in the child reported here. Similarly, generalised joint hypermobility, a prominent feature of EDS types I to III and V to VIII, was not observed in this child except for mild hypermobility in his elbows, wrists, and feet.

The level of the amino-terminal propeptide of type III procollagen in the serum has been reported to be low in patients with EDS IV. ${ }^{9}$ The propeptide is normally cleaved from the procollagen in the extracellular processing of procollagen to collagen. It has been proposed that some of the released propeptide enters the circulation and that its level can be used as an indicator of the level of type III collagen synthesis. ${ }^{9}$ The serum assay was normal in this child and would, therefore, not have been useful as a screening test for EDS IV. The normal serum level of the propeptide was not expected in view of the low amounts of type III collagen in the dermis and the low production and secretion of type III collagen by cultured fibroblasts. ${ }^{7}$

Increased susceptibility to bruising, vascular fragility and gastrointestinal abnormalities occur in many forms of EDS but they are major features of EDS IV. ${ }^{1}$ In the proband, bleeding from the large intestine appeared to be because of fragility of the fine connective tissue stroma of the mucosa and mucosal vessels. There did not appear to be a major abnormality in platelet function or coagulation to account for the mucosal bleeding and bruising. However, the skin bleeding time was mildly increased which could have been the result of increased vascular fragility or an abnormality in the ability of the subintimal collagen to stimulate platelet aggregation. The child's platelets aggregated normally when exposed to normal collagen but there were insufficient amounts of mutant type III collagen available to enable these in vitro assays to be repeated with mutant collagen. However, it is likely that the mutant type III collagen would have reacted normally with platelets as the normal reactive domain is in the middle of the $\alpha$ (III) chain and not in the inactive carboxy-terminal region that contained the deletion. ${ }^{11}$

He was also constipated and gastric emptying was delayed. The mechanisms involved in these anomalies of intestinal motility were not investigated but they were likely to be the result of the abnormal connective tissue structure of the bowel wall.

The skin was clinically fine and thin in keeping with our previous finding that its type III collagen content had been reduced to $11 \%$ of normal. ${ }^{7}$ The collagen fibrils were round in cross section and there was an essentially unimodal distribution of fibril diameters with occasional small fibrils. It is likely that type I and III collagens normally occur together in dermal fibrils. ${ }^{12}$ However, the mean fibril diameter in the proband's thigh dermis was not significantly reduced when compared to control samples from the inguinal region. These results were difficult to interpret because variations in fibril diameter have been observed in normal samples from different sites. ${ }^{13}$ Others have reported reduced collagen fibril diameters and heterogeneity in fibril distribution with some large and irregular fibrils. ${ }^{1314}$

The reduced amount of type III collagen in the dermis from the proband was probably the result of decreased synthesis and secretion of type III collagen by the fibroblasts as observed in vitro. However, the rough endoplasmic reticulum of dermal fibroblasts was not distended as has been observed in other cases of EDS IV. ${ }^{315}$ In some of the latter cases, immunofluorescent studies have shown accumulation of type III collagen in the cisternae of the rough endoplasmic reticulum. ${ }^{15}$

Our previous results showed that mutant homotrimeric molecules were the major triple helical species of type III collagen produced by cultured dermal fibroblasts. ${ }^{7}$ They had a normal thermal denaturation temperature which suggested that the shortened molecules were able to form a stable triple helix. However, despite the apparent stability of these molecules and their resistance to mammalian collagenase digestion, type III collagen did not accumulate in the dermis. It appears that this outcome was prevented by the low production and secretion of type III collagen.

The normal distribution of type III collagen in the vascular system, dermis, and in the supporting connective tissues of the viscera is consistent with the clinical features of EDS IV expressed by the proband. ${ }^{1}$ It also explains the susceptibility of EDS IV patients to other manifestations not shown by the proband, for example, mitral valve prolapse and aneurysms and rupture of major arteries such as the aorta and of medium sized arteries such as the splenic, renal, popliteal, and carotid arteries. ${ }^{116}$

The proband was heterozygous for the point mutation at the splice donor site of intron 41 of the COL3A1 gene. ${ }^{7}$ In the present study, only normal genomic DNA sequences were obtained after amplification and sequencing of leucocyte DNA from the parents. It is likely, therefore, that the proband had a new dominant mutation, but gonadal mosaicism in one parent was not excluded as an alternative mechanism. The heterozygous mutation of type III collagen in the proband with EDS IV provides an explanation for his clinical phenotype. From our findings and other reports, it appears that the acrogeric type of EDS IV can arise from deletions or amino substitutions within the triple helical domain of $\alpha 1$ (III) chains..$^{14-19}$

The authors express their appreciation to $\mathrm{T}$ Dibbayawan and $\mathrm{A}$ Davies for assistance with the electron microscopy, Professor N Buchanan for referring the patient, and $\mathrm{Dr} \mathrm{C}$ Ollie for collection of a 
blood sample. The work by $\mathrm{D} O \mathrm{O}$ Sillence was supported by the Vallack bequest (University of Sydney) and the research by $W$ G Cole and A Chiodo was supported by the National Health and Medical Research Council of Australia and the Royal Children's Hospital Research Foundation, Melbourne.

1 Pope FM, Nicholls AC, Narcisi P, et al. Type III collagen mutations in Ehlers-Danlos syndrome type IV and other related disorders. Clin Exp Dermatol 1988;13:285-302.

2 Pope FM, Nicholls AC, Jones PM, Wells RS, Lawrence DJ. EDS IV (acrogeria): new autosomal dominant and recessive types. F $R$ Soc Med 1980;73:180-6.

3 Byers PH, Holbrook KA, McGillivray B, MacLeod PM, Lowry RB. Clinical and ultrastructural heterogeneity of type IV Ehlers-Danlos syndrome. Hum Genet 1979;47:141-50.

4 Cikrit DF, Miles JH, Silver D. Spontaneous arterial perforation: the Ehlers-Danlos specter. $\mathscr{f}$ Vasc Surg 1987;5:248-55.

5 Lach B, Nair SG, Russell NA, Benoit BG. Spontaneous carotid-cavernous fistula and multiple arterial dissections in type IV Ehlers-Danlos syndrome. $\mathcal{f}$ Neurosurg 1987;66:462-7.

6 Pope FM, Nicholls AC, Dorrance DE, Child AH, Narcisi P. Type III collagen deficiency with normal phenotype. $\mathcal{F} R$ Soc Med 1983;76:518-20.

7 Cole WG, Chiodo AA, Lamande SR, et al. A base substitution at a splice site in the COL $3 A 1$ gene causes exon skipping and generates abnormal type III procollagen in a patient with Ehlers-Danlos syndrome type IV. I Biol Chem 1990; 265:17070-7.

8 Cole WG, Evans R, Sillence DO. The clinical features of Ehlers-Danlos syndrome type VII due to a deletion of 24 amino acids from the pro- $\alpha 1$ (I) chain of type I procollagen. $\mathcal{f}$ Med Genet 1987;24:698-701.

9 Dyne KM, Zanaboni G, Annoni G, De Agostini MP, Cetta G. Low levels of serum type III procollagen aminoterminal propeptide confirmed type III collagen deficiency in patients without typical clinical symptoms of Ehlers-Danlos type IV. Eur f Clin Invest 1989;19:362-6.

10 Beighton P, Price A, Lord J. Variants of the Ehlers-Danlos syndrome. Clinical, biochemical, haematological, and chromosomal features of 100 patients. Ann Rheum Dis 1969;28:228-42.

11 Morton LF, Fitzsimmons CM, Rauterberg J, Barnes MJ. Platelet-reactive sites in collagen. Collagens I and III possess different aggregatory sites. Biochem $\mathcal{F}$ 1987;248:483-7.

12 Keene DR, Sakai LY, Bachinger HP, Burgeson RE. Type III collagen can be present on banded collagen fibrils regardless of fibril diameter. 7 Cell Biol 1987;105:2393-402.

13 Davies A, Dibbayawan T, Dunlop H, Sillence D. Collagen microfibril aggregation in normal human inguinal skin. Connect Tissue Res (in press).

14 Superti-Furga A, Gugler E, Gitzelmann R, Steinmann B. Ehlers-Danlos syndrome type IV: a multi-exon deletion in one of the two COL $3 \mathrm{~A} 1$ alleles affecting structure, stability and processing of type III procollagen. $\mathcal{f}$ Biol Chem 1988;263:6226-32.

15 Byers PH, Holbrook KA, Barsh GS, Smith LT, Bornstein MD. Altered secretion of type III procollagen in a form of type IV Ehlers-Danlos syndrome. Lab Invest 1981;44:336-41.

16 Clark JG, Kuhn C, Uitto J. Lung collagen in type IV EhlersDanlos syndrome: ultrastructural and biochemical studies. Am Rev Respir Dis 1980;122:971-8.

17 Tromp G, Kuivaniemi H, Shikata H, Prockop DJ. A single base mutation that substitutes serine for glycine 790 of the $\alpha$ l(III) chain of type III procollagen exposes an arginine and causes Ehlers-Danlos syndrome IV. J Biol Chem 1989;264:1349-52.

18 Tromp G, Kuivaniemi H, Stolle C, Pope FM, Prockop DJ. Single base mutation in the type III procollagen gene that converts the codon for glycine 883 to aspartate in a mild variant of Ehlers-Danlos syndrome IV. $\mathcal{f}$ Biol Chem 1989;264:19313-7.

19 Kuivaniemi H, Kontusaari S, Tromp G, Zhao M, Sabol C, Prockop DJ. Identical $\mathrm{G}^{+1}$ to $A$ mutations in three different introns of the type III procollagen gene (COL $3 A 1)$ produce different patterns of RNA splicing in three variants of EhlersDanlos syndrome IV. $f$ Biol Chem 1990;265:12067-74. 